then subjected to carbon and oxygen isotope ratio measurements using standard procedures. The results were, of course, numerical in detail, but the general picture is easily sketched. The data revealed distinct isotopic trends with all examples of each component falling within a distinct and limited field.

That such systematic information can be obtained at all emphasizes the importance of taking an inhomogeneous rock apart and analysing the components separately. Failure to do this in the past has often produced fuzzy data with large variations liable to misinterpretation. That such systematic information was actually obtained in this case, however, indicates that no major re-equilibration has occurred since the dolomite was laid down during the Precambrian. Since that time there has been no extensive interaction with meteoric waters, no deep burial, no more than minimal metamorphism and little deformation.

All the indications are, therefore, that the isotopic ratios and trends are original. What's more, those ratios and trends bear a striking similarity to those from primary Phanerozoic limestones. Tucker thus concludes that the Beck Spring Dolomite and, by implication, the many other Precambrian dolomite rocks with comparable properties were precipitated directly as dolomites and thus not generated by the dolomitization of directly precipitated limestones. And as the most important control on carbonates is the isotopic composition of the water from which they are precipitated, the inevitable, or at least the simplest, overall conclusion is that the Precambrian seawater that favoured the production of $\mathrm{Ca}-\mathrm{Mg}$ carbonates (dolomites) must have been chemically different from the Phanerozoic seawater that gave, and gives, rise to $\mathrm{Ca}$ carbonates (limestones).

Unfortunately, Tucker does not elaborate on the difference, except to note the general consensus that the seawater characteristics favouring dolomite precipitation would include a higher than usual $\mathrm{Mg} / \mathrm{Ca}$ ratio, a higher $\mathrm{CO}_{2}$ partial pressure, a higher temperature and a lower $\mathrm{SO}_{4}{ }^{2-}$ content. So mysteries remain. Why does dolomite not precipitate from modern seawater if, as Tucker claims, it is favoured thermodynamically? Why have experiments to synthesize dolomite under simulated sedimentary conditions merely resulted in an imperfectly ordered, Ca-rich version of dolomite ('proto-dolomite')? Why, if dolomite was the preferred precipitate during the Precambrian, were limestones nevertheless sometimes generated? And when (and why) precisely did the preference change from dolomite to limestone?

What the answers to these questions might say about dolomite is of comparatively minor importance, but what they might say about the evolution of seawater composition could be a rather bigger issue. $\square$

\title{
Intermediate valence compounds as applicable materials?
}

\section{from Michael Kelly}

UNDER modest pressures, the normally semiconducting samarium sulphide undergoes a first-order phase transition to a metallic state. Thus by rubbing or scribing the surface of this material, an intrinsic metal-semiconductor contact can be generated and can subsequently be removed by temperature excursions of approximately $100^{\circ} \mathrm{C}$.

Possible applications of this and other surprising properties of the so-called intermediate valence compounds, especially those involving compounds and alloys of the rare-earth elements, were outlined by $\mathbf{P}$. Wachter (Zurich) in the closing session of a one-day meeting* devoted to the physical properties of the these materials. As the name implies, the ionic constituents of intermediate valence compounds may carry non-integral charges. Briefly, in the solid environment, only a small energy difference may be involved in the transfer of an electron from an incomplete $f$ shell of a rare-earth atom to the available states of the valence band. The result is that there may be dramatic anomalies of physical properties not so far explained by conventional valence band theory.

Wachter opened the meeting with a review of recent work by his team on semiconducting intermediate valence compounds. Because of the weak interactions of electrons in the f shells of adjacent rare-earth atoms, the characteristic energy gaps are of order 10 $\mathrm{meV}$, corresponding to temperatures of order $100 \mathrm{~K}$. These are similar to energies associated with magnetic ordering and farinfrared optical absorption, so that small temperature changes can induce substantial changes in many physical properties. The metal-insulator transition under pressure is accompanied by a volume change of order 10 per cent, because of the different size of the rare-earth atom in its different charge states. As an incipient instability, it causes the elastic properties to be anomalous under ambient conditions. Compressibilities are enhanced, some elastic constants are negative and finite wavelength vibrational instabilities are encountered. G. Saunders (University of Bath) reported on these anomalies as the transition is approached. Possible applications as pressure sensors and phonon generators can be envisaged.

The rare-earth metals and their alloys (including those exemplified by $\mathrm{Eu} \mathrm{Cu}_{2} \mathrm{Si}_{2}$ ) have optical, magnetic and electronic

*The meeting was held at the Long-Range Research Laboratory at the GEC Hirst Research Centre, East Lane, Wembley, UK, on 15 January 1982. transport properties that vary as the valence changes (reviewed by $D$. Wohlleben, University of Koln, and F. de Boer, University of Amsterdam). The valence changes generally take place at low temperatures, so that in ambient conditions most physical properties, though anomalous, are 'quiet'. The metallic alloys are easier to prepare, are simpler to analyse and are proving a fertile testing ground for the current theories of intermediate valence phenomena.

Preparation of pure and stoichiometric sulphides of rare-earth metals is proving difficult (H. Bach, University of Bochum), especially because of the high volatility of sulphur with respect to the rare-earth metals.

The low-temperature electrical conductivity of $\mathrm{SmB}_{6}$, and its sample dependence in particular, pose unanswered questions that impinge on the transport theories of disordered systems (Sir Nevill Mott, University of Cambridge). The magnitude of the resistivity of TmS and its relative temperature independence below $1 \mathrm{~K}$ is a further puzzle. Attempts to extract an effective one-electron theory from the general many-electron formulation, in order to account for the anomalies in lowtemperature specific heat and magnetic susceptibility of the metals, were reviewed by D. Newns (Imperial College). Mixed valence materials are those where one species is present in one of two charge states, and they have already found applications, particularly as dyes. P. Day (University of Oxford) cited many other examples, including $\mathrm{Fe}_{3} \mathrm{O}_{4}$ (magnetite) and $\mathrm{LiTi}_{2} \mathrm{O}_{4}$ (which goes superconducting).

Other points raised in the open-ended discussion included the inability of the compounds to survive more than ten cycles through the pressure-induced phase transition without damage, and the fact that doping (apart from alloying in metals) has not been investigated, as nonstoichiometry is already providing a wealth of phenomena. Catalytic properties have not been examined but were the subject of speculation.

Given our increased understanding of the semiconducting intermediate valence compounds, it remains for further advances in the methods of preparation to be achieved, and a wider characterization undertaken, before they replace existing materials in device applications or forge a new range of devices based on their unique combination of physical properties.

Michael Kelly is at the Hirst Research Centre of the General Electric Company Limited, Wembley, Middlesex HA9 $7 P P$. 